\title{
Bitcoin: The Road to Hell Is Paved With Good Promises
}

\author{
Sofoklis Vogiazas* ${ }^{*}$ Constantinos Alexiou ${ }^{\dagger}$
}

\begin{abstract}
In this paper, by using econometric techniques we provide evidence that bitcoin exhibited the formation of speculative bubble in 2017. To conceptually rationalize the results, we delve into the extant theoretical approaches developed by Kindleberger's (1978) speculative bubbles and Minsky's (1992) financial instability hypothesis. Certainly, bitcoin has spurred a revolution in payment technology that, if treated cautiously can facilitate financial intermediation and inclusion. Ultimately, whether or not bitcoin constitutes a bubble is a decision for investors as the road to hell is paved with good promises.
\end{abstract}

(J.E.L.: B26, C22, E4, G1).

\section{Introduction}

Undoubtedly the world of money and finance is transforming ever so dramatically. The novel digitized assets and innovative channels have ushered in a new era in the realm of financial transactions and alternative forms of capital. The key characteristic of the so-called cryptocurrencies lies in the very fact that is shared between network participants, adopting the distributed ledger technology and using native tokens as means of facilitating the process in the absence of a central authority or any regulation.

Bitcoin, a stateless cryptocurrency built on revolutionary blockchain technology, is a digital, global and decentralized form of money designed to allow people to exchange value directly bypassing traditional central clearinghouses. Although bitcoin has no intrinsic value or claim to the issuer, it can be traded, invested in, and even developed.

Bitcoin became the fastest-growing asset in the world in 2017. This momentum coupled with an increasing betting by speculators on how far it can rise has spawned fears for an imminent formation of an economic bubble. In this context, economists have been swift to compare bitcoin's stratospheric momentum with past bubbles such as the 17th century's tulip

\footnotetext{
*Black Sea Trade and Development Bank, Thessaloniki, Greece. E-mail: svoyazas@gmail.com

${ }^{\dagger}$ Cranfield School of Management, Cranfield University, UK. E-mail: constantinos.alexiou @ cranfield.ac.uk
} 
mania or even the dotcom bubble that eventually burst in 2000. Not long time ago, Oliver White at Fathom Financial Consulting observed that bitcoin possesses all characteristics of a bubble asset. By plotting the price of bitcoin against its historical average and other mainstream assets they found that the current value of bitcoin is six times its average price since 2013.

The history of financial markets is littered with asset price bubbles. Although, the underlying rationality of driving bubbles may be fascinating, yet is predictable and fundamentally flawed. In the case of bitcoin, a perpetual price rise will mean that the value of bitcoin must at some point exceed all of the world wealth. The Ponzi scheme theory predicts that the scheme will collapse unless the irrational belief in the next fool holds. In his analysis, Minsky (1992) identified three types of borrowers out of which one was referring to the 'Ponzi borrower' whose behaviour feeds on the belief that the appreciation of the value of the asset will be sufficient to refinance the debt, hence, keeping the Ponzi borrower afloat. The existence of Ponzi-style finance in the financial system creates a bubble which bursts when the asset prices cease to increase. The domino effect that follows causes speculative borrowers to collapse, thereby, unleashing a destructive wave that adversely affects hedge borrowers. In other words, the irrationally exuberant Ponzi investors cause the market to crash.

In view of the bitcoin bubble-saga by utilizing the recursive procedures advanced by Phillips et al. $(2011,2015)$ we provide evidence on the empirical validity of the bubble assertions that are making headlines globally. At the same time, by drawing on the seminal works of Kindleberger (1978) and Minsky (1992), we provide a platform upon which a comprehensive analysis is established in the realm of cryptocurrencies. We argue that the extant structure of the financial system, devoid of an effective regulatory framework, has been fuelling the surging price of bitcoin. We, therefore, feel that the proposition put forward by Minsky and Cambell (1987) to design a new effective regulatory framework which will be reassessed frequently and also be consistent with evolving markets and financial structures, is needed. Although regulators have a duty to protect investors, they seem to be at odds over how to regulate the cryptocurrency universe. Certainly, cryptocurrencies are difficult to regulate as they operate on a global scale, being opaque and outside the conventional financial system. Yet, as Kregel (2014) very eloquently put it 'after cases of severe disruption, regulations are adapted to prevent the occurrence of crises that have already occurred' (p. 220).

In the following section, we provide a brief overview of the literature and in section 2 we describe the methodological framework. Section 3 presents our results and discusses them in the prism of Kindleberger (1978) and Minsky (1992) insight that is considered particularly relevant in the case of bitcoin whilst section 4 concludes. 


\section{Brief Literature Review}

Although the empirical evidence on cryptocurrencies remains scarce, a number of studies have emerged on the bitcoin's bubbly nature. More specifically, Gomez-Gonzalez and Parra-Polania (2014) create a demandsupply model which shows that a significant part of asset price volatility can be related to the volatility of noise in the signals. The authors suggest that in the case of bitcoin it is expected that the noise volatility may be much higher since it is not clear what is the reference information related to the fundamental value. Overall, the absence of an intrinsic value represents a problem for the stability of bitcoin's price as an asset. Hence, in the absence of fundamental factors, a rational expectations equilibrium for the price is not guaranteed (Gomez-Gonzalez and Parra-Polania, 2014).

Yermack (2014) suggests that the zero correlation of bitcoin with widely used currencies and gold makes it useless for risk management and exceedingly difficult for its owners to hedge. In this regard, bitcoin behaves more like a speculative investment vehicle than a currency (Yermack, 2014).

MacDonell (2014) uses an autoregressive moving average model (ARMA) to explain trading values and then applies a log-periodic power law (LPPL) model in an attempt to predict crashes. The results of ARMA modelling show that bitcoin values react to the CBOE volatility index (VIX), suggesting that a primary force driving bitcoin values is speculation by investors whereas the LPPL model accurately predicts ex-ante the crash that occurred in December 2013. In the same vein, Sornette and Cauwels (2014) analysis of the acceleration in bitcoin's price growth rate using the hyperbolic growth model, enables them to diagnose that bitcoin was in a bubble in November 2013 and that a correction would come when the market entered a critical state as a result of positive feedback mechanisms.

Using the Granger causality framework applied in two relationships (bitcoin price and trade transactions; bitcoin price and investors' attractiveness), Bouoiyour et al. (2015) confirm the speculative nature of bitcoin, yet without overlooking its usefulness in economic reasons. In another study, Bouoiyour and Selmi (2015) by using the ARLD bounds testing method find no sign of bitcoin being a safe haven but more of a speculative foolery.

Baek and Elbeck (2014) study on the economic variables that drive the returns of bitcoin provides evidence that its volatility is internally driven, leading to the conclusion that the bitcoin's market is highly speculative. In a similar tone, Baur et al. (2017) provide evidence that bitcoin is uncorrelated with traditional asset classes such as stocks, bonds and commodities both in normal times and in periods of financial turmoil. Through the analysis of transaction data, the authors suggest that bitcoin is used as a speculative investment and not as a medium of exchange. 
Following MacDonell (2014), Cheah and Fry (2015) study in the period January 2013 to November 2013 determines that the crash in December 2013 was preceded by a bubble. In a comprehensive review of methods and tools used in modelling bitcoin's price, Fantazzini et al. (2016) conclude that the key drivers of bitcoin price dynamics are mainly of speculative nature, followed by traditional supply and demand-related variables, while the global macro-financial variables play no role. Cochrane (2017) explains the price surge in bitcoin as a 'normal' phenomenon in financial markets, described as the intersection of a convenience yield with speculative demand and a temporarily limited supply, plus a temporarily limited supply of substitutes and limits on short-selling. This phenomenon is further supported by a lot of asymmetric information or opinion to spur trading, and the shady source of bitcoin demand (Cochrane, 2017). The results of Kroeger and Sarkar (2017) analysis indicate that price uncertainty inhibits the use of bitcoin as a store of value, as the authors provide evidence on the existence of persistent, statistically significant differences between bitcoin prices in multiple USD exchanges.

\section{Methodological Approach}

Our dataset consists of the closing prices for the bitcoin price index in US Dollar terms ${ }^{1}$ over the period July 2010 to December 2017. Figures 1 and 2 show bitcoin prices from 18 July, 2010 to 31 December, 2014, and from 1 January, 2015 to 4 December, 2017, respectively. From Figure 1 it seems that bitcoin's price during this period was relatively stable before peaking dramatically in late 2013. Trading for about $\$ 13.5$ at the beginning of 2013, it soared to more than $\$ 1200$ before falling by about 50 per cent in December 2013 after China's central bank barred financial institutions from handling bitcoin transactions. In early 2014, bitcoin's price plunged again when one of the biggest trading platforms at that time filed for bankruptcy protection after being beset for security breaches. However, as shown in Figure 2, the price followed a mildly upward trend until the beginning of 2017 when a steep rise is evident. In September 2017, the price of bitcoin plummeted for days as China decided to close down the country's bitcoin exchanges. Since then the price of bitcoin recovered, then fell again when South Korea announced that it would ban raising funds through all forms of virtual currencies.

Overall, bitcoin exhibits an extremely volatile pattern as it has declined by more than 50 per cent on eight separate occasions since 2011 which reflects a material exchange risk and limits bitcoin's ability to serve as a reliable source of value.

${ }^{1}$ Sourced from http://www.coindesk.com/price/ 


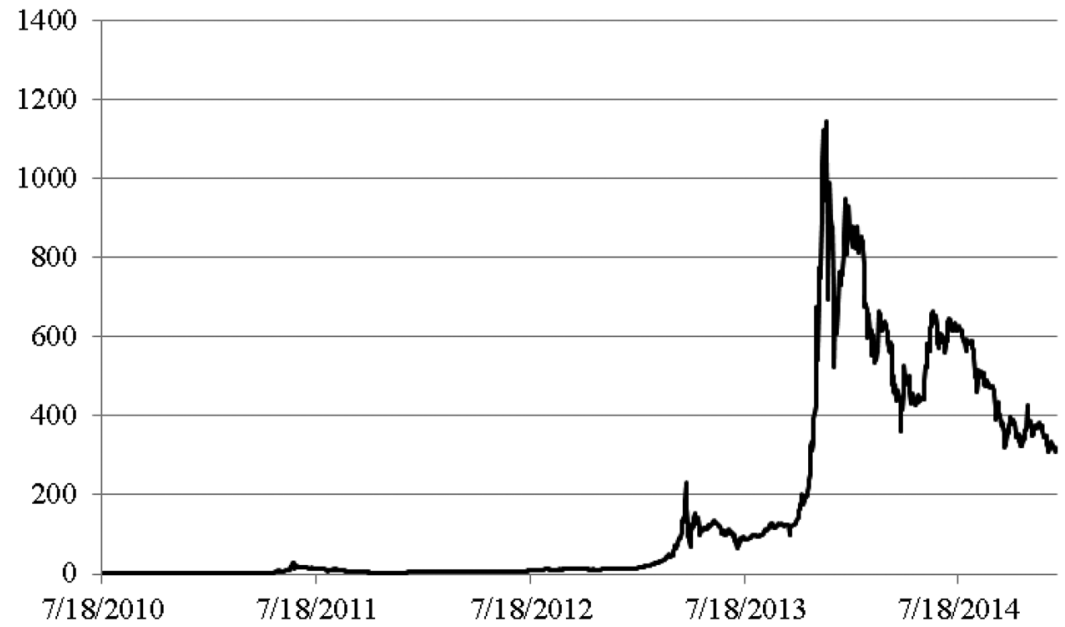

Figure 1: Daily Closing Prices of Bitcoin in 18/07/2010-31/12/2014 (US Dollars)

In detecting and date-stamping price bubbles in bitcoin, we employ the tests developed by Phillips et al. (2011, hereafter SADF test) and Phillips et al. (2015, hereafter GSADF test) which are powerful test procedures that exploit this feature of explosiveness to identify bubbles. The detection strategy is based on a right-tail variation of the standard Augmented Dickey-Fuller (ADF) unit root test wherein the null hypothesis is of a unit root and the alternative is of a mildly explosive process. Rejection of the

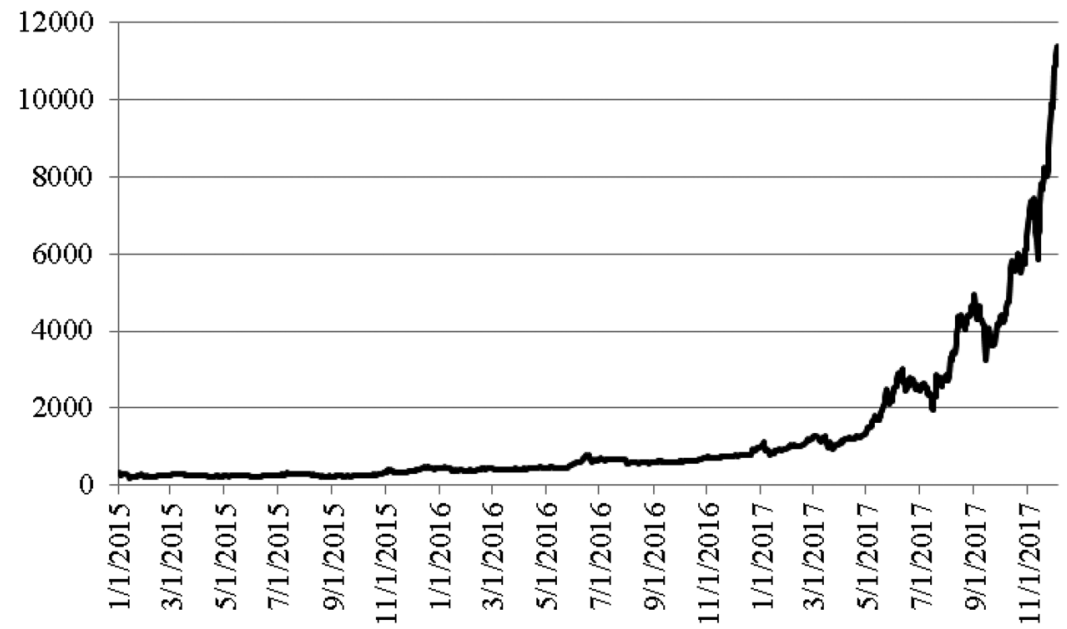

Figure 2: Daily Closing Prices of Bitcoin in 01/01/2015-4/12/2017 (US Dollars) 
null in these two tests may serve as empirical evidence for an asset price bubble, and in the case of the SADF and GSADF tests, enables us to datestamp its occurrence. Phillips et al. (2011) and Phillips et al. (2015) have shown that the recursive and rolling tests yielded higher power in the detection of bubbles, compared to standard tests. Both test strategies employed (SADF, GSADF) are based on some variation of the following reduced form empirical equation:

$$
y_{t}=\mu+\delta y_{t}+\sum_{i=1}^{p} \varphi_{t} \Delta y_{i-1}+\varepsilon_{t}
$$

where $y_{t}$ is the price of bitcoin, $\mu$ is an intercept, $\mathrm{p}$ is maximum number of lags, $\pi_{i}$ for $i=1 \ldots p$, are the differenced lags coefficients and $\varepsilon_{t}$ is the error term, where $\varepsilon_{\mathrm{t}} \sim$ iid $\left(0, \sigma^{2}\right)$. Testing for a bubble (explosive behaviour) is based on a right-tail variation of the standard ADF unit root test where the null hypothesis is of a unit root and the alternative is of a mildly explosive autoregressive process (Phillips and Magdalinos, 2007). Formally, we test for:

$$
\begin{aligned}
& \mathrm{H}_{\mathrm{o}}: \delta=1 \\
& \mathrm{H}_{1}: \delta>1
\end{aligned}
$$

It should be noted that the GSADF test utilizes a recursive flexible window method that is better suited for practical implementation with time series delivering a consistent real-time stamping strategy for the origination and termination of multiple bubbles. Hamilton (1986) suggests that the interpretation of tests for speculative price bubbles depends on the nature of any non-stationarity in the fundamentals. Thus, rejection of the 'no-bubble' null that implies the acceptance of the presence of a bubble is only possible within the specified model.

\section{Empirical Results and Discussion}

The tests and critical values simulations are performed using the routines described in Caspi (2017). The optimal lag length is chosen by the Schwartz Information Criterion (SIC) when estimating equation (1) for the whole sample (with the maximum number of lags set to 12). Accordingly, the lag length in the recursive procedure is set to 3 . The SADF statistic is recursively estimated with an initial window size of 36 observations as the data have been converted to monthly. The choice of initial window size relies on Phillips et al. (2011) and Phillips et al. (2015) who use a window size of about 3 years for monthly data. Although the choice of minimal 
window size is arbitrary, our results are robust to different choices of window sizes and lags.

Figures 3 and 4 and Table 1 present the results obtained from the SADF and GSADF tests in the sample period 2010-2017. Earlier contributions have dealt with the period prior to 2015. A case in point is the study by Malhotra and Maloo (2014) who provide evidence of explosive behaviour in the bitcoin to USD exchange rate during November 2013 to February 2014. Our results are in line with the studies of Cheah and Fry (2015) and Malhotra and Maloo (2014). Furthermore, our results from both tests suggest that around the first quarter of 2017, bitcoin seemed to be heading in a classic bubble territory. In other words, by rejecting the null hypothesis of a unit root, the results from both tests provide significant evidence of a speculative bubble in bitcoin's price which begins around the first quarter of 2017.

Phillips et al. (2015) suggest that the use of these tests over long historical periods presents a serious econometric challenge due to the complexity of the nonlinear structure and structural-break mechanisms that are inherent in multiple-bubble phenomena within the same sample period. As a robustness test, we have split the bitcoin's time series into two subperiods; the results proved to be practically unaffected by the frequency or length of time series.

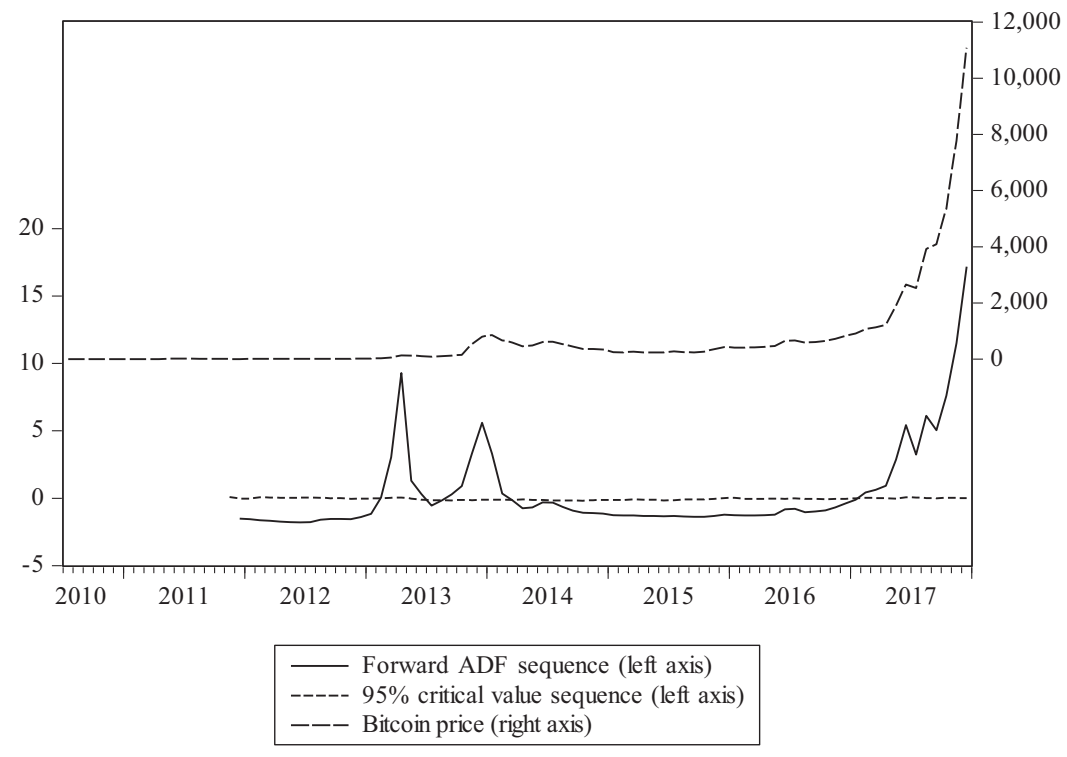

Figure 3: SADF Test (July 2010-Dec 2017) 
8 Economic Notes 9999-2018: Review of Banking, Finance and Monetary Economics

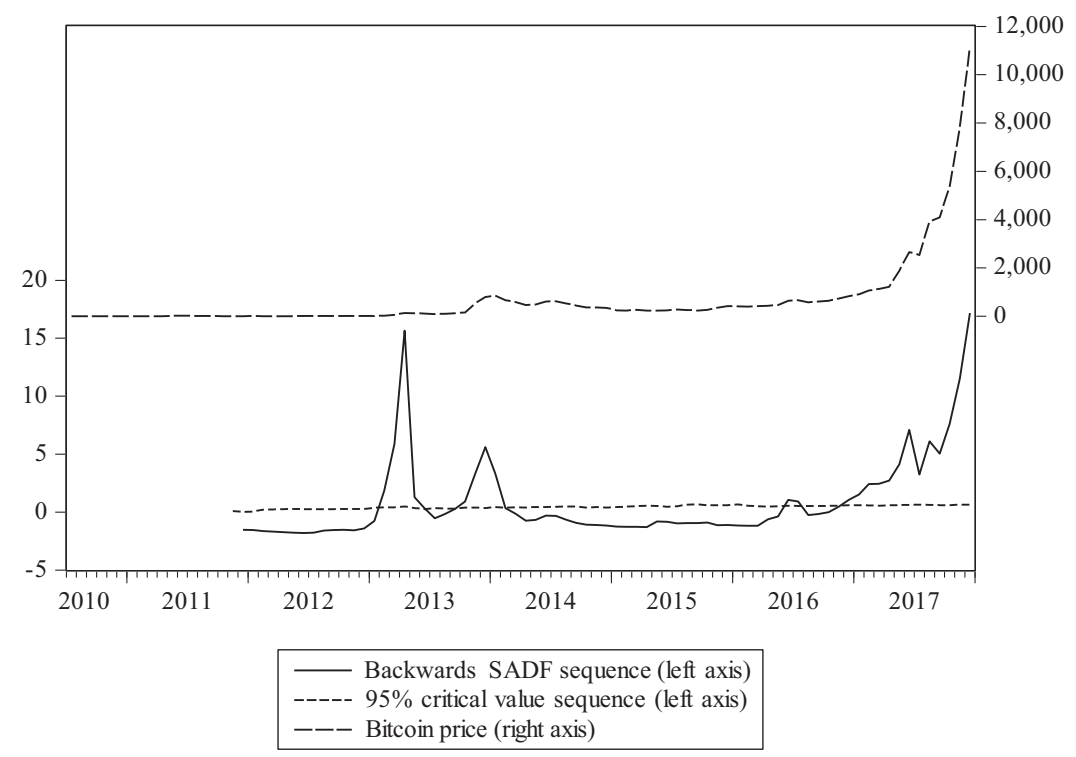

Figure 4: GSADF Test (July 2010-Dec 2017)

Although our results indicate the presence of bubble formation in bitcoin, the question of what has caused the burgeoning effect on its price deserves particular attention. Is the surge in the price of bitcoin purely speculative or, as the Economist ${ }^{2}$ nicely puts it, is it evidence that bitcoin deserves a more substantial role as a medium of exchange or a store of value?

To conceptually rationalize the yielded evidence, we delve into the extant theoretical approaches developed by Kindleberger's (1978) work on speculative bubbles and Minsky's (1992) financial instability hypothesis. In this context, the key stages that an asset has to go through before becoming a bubble are: displacement, credit creation, euphoria, financial distress and revulsion.

According to the Minskian approach, capitalist economies are inherently unstable in a sense that business cycles do not depend on

Table 1: Results of the Date-Stamping Procedures

\begin{tabular}{lcc}
\hline Period No & SADF & GSADF \\
\hline 1 & 2013:M3-2013:M6 & 2013:M1-2013:M6 \\
2 & 2013:M9-2014:M2 & 2013:M10-2014:M1 \\
3 & 2017:M2- & 2016: M12- \\
\hline
\end{tabular}

\footnotetext{
${ }^{2}$ The Economist (3 June, 2017) What if the bitcoin bubble bursts?
} 
exogenous shocks but are contingent on the internal compounding dynamics of the economies as well as the extant regulatory framework designed to keep the economy operating within reasonable bounds. The underlying principles of the financial instability hypothesis can be viewed as an interpretation of Keynes's General Theory as well as the credit view of money and finance by Schumpeter (1934), and the seminal works of Minsky (1975, 1986). In particular, Minsky (1992) in his attempt to expound upon the financial instability hypothesis argued that:

"Over a protracted period of good times, capitalist economies tend to move from a financial structure dominated by hedge finance units to a structure in which there is large weight to units engaged in speculative and Ponzi finance. Furthermore, if an economy with a sizeable body of speculative financial units is in an inflationary state, and the authorities attempt to exorcise inflation by monetary constraint, then speculative units will become Ponzi units and the net worth of previously Ponzi units will quickly evaporate. Consequently, units with cash-flow shortfalls will be forced to try to make position by selling out position. This is going to lead to collapse of asset value" (p. 8).

The setting for the development of the Minskian hypothesis is given by the self-sustaining dis-equilibrating processes observed by Kindleberger (1978) in past bubbles. This theory builds upon the immature, fraud-prone markets which provide an ideal setup for the built up of a Ponzi scheme thanks to humans' tendency to herd behaviour.

"What happens, basically, is that some event changes the economic outlook. New opportunities for profit are seized and overdone, in ways so closely resembling irrationality as to constitute a mania. Once the excessive character of the upswing is realized, the financial system experiences a sort of "distress", in the course of which the rush to reverse the expansion process may become so precipitous to resemble panic. In the manic phase, people of wealth or credit switch out of money or borrow to buy real or illiquid assets. In panic, the reverse movement takes place, from real or financial assets to money, or repayment of debt, with a crash in the prices of commodities, houses, buildings, land, stocks, bonds-in short, in whatever has been the subject of the mania" (Kindleberger, 1978, p. 5).

Herding behaviour drives non-professional investors to join the market insiders in a euphoric buying spree as expectations become over-optimistic during the phase of bubble making. As expectations are not stable over time when the bubble approaches its peak, they suddenly reverse to overpessimism. As a consequence of this change in expectations, investors rush to sell and the market collapses.

The herding behaviour which suggests a coordinated departure from rationality is a key ingredient in Kindleberger's (1978) theory. Though the 
dynamic evolution of expectations, the culmination of a period of expansion leads to a downturn. Expectations become over-optimistic during the phase of bubble formation and lead to overinvestment in the market affected by these expectations. Since expectations are not stable over time and driven by the so-called 'animal spirits' when the bubble approaches its peak, expectations reverse to over-pessimism, often this happens suddenly. As a consequence of this change in beliefs, investor rush to sell their investment and the market collapses. As in the Great Depression, it all starts from a mania that implies a loss of touch with reality, something close to mass hysteria (Kindleberger, 1973). Thus, coordinated departures from rationality occur which eventually lead to the rise and then to the burst of bubbles.

Another critical ingredient, inspired by Minsky (1992), is the interaction among heterogeneous agents. Kindleberger identifies two main groups of agents: insiders and outsiders. While the well-informed insiders move first, the outsiders follow and usually are the ones who lose the most during the bubble burst. Typically, outsiders buy when prices are already high because they expect the prices to rise even more. As the bubble approaches its peak, investors' behaviour based on irrational expectations becomes more common. The euphoria is widespread and investors behave almost as if they expect the prices to increase perpetually. A bubble often starts with an exogenous shock, a displacement in Kindleberger's words. This shock could be induced by a change in the financial instrument, a sudden accessibility of a new market, a technological innovation or a change in the laws or regulation shaping the investment landscape. And it is much easier to setup Ponzi schemes in an over-optimistic environment, especially when lack of understanding or financial illiteracy reign (Haldane, 2017).

Yet, a crucial point in Kindleberger's work is the absence of a 'lender of last resort'. In bitcoin's case, we are of the view that perhaps now is the right time for the 'lender of last resort' to step in. Certainly, cryptocurrencies offer potential benefits, including speed and efficiency in making payment and promoting financial intermediation and inclusion (He et al., 2016). At the same time, cryptocurrencies pose substantial risks to the financial system which is becoming increasingly entangled with them.

On a different note, the Marxist perspective is more towards the usevalue of a currency. In this context, it can be argued that when treating tokens as currency then those tokens will have a useful function only if they are accepted as a valid currency. In other words, those tokens have a usevalue only if they have an exchange value. What is remarkable about bitcoin is that despite that fact that it barely constitutes a currency, yet it is widely used. The salient question that arises is, therefore, why is this so? It could be argued that this is a typical case where a speculative bubble is brewing. Being devoid of an economic anchor, bitcoin is exposed to increasing bouts 
of speculation that may trigger a snowball effect, hence pushing its price up. Of course, the inherent susceptibility of bitcoin suggests that its price can potentially collapse abruptly. Episodes of the hacking behaviour of other malfunctions have caused bitcoin prices to be adversely affected, causing investors to abandon the cruise hence wiping out fortunes in the process. Investing in bitcoin is, therefore, a highly risky but nevertheless profitable way to make gains in a short period of time.

Currently, banknotes and electronic transfers clearly are not convertible to gold or backed by any form of a physical commodity. The currency is directly related to the ability of any government to guarantee its validity as well as the performance of the economy per se. Clearly, bitcoin aims to serve as a medium of exchange, but it is disjointed from any governmental control or tied to the performance of the economy. Being devoid of an economic anchor the value of bitcoin is left to the whims of speculative investors. It is, therefore, a currency permeated with unsurmountable contradictions. It is utterly unthinkable to drive a wedge between money and societies that use it every day in their economic activities. This is after all the very reason, why central banks came into existence in the first place, that is, to make all commodities convertible having a common denominator. The whole rationale behind adopting bitcoin as a currency is a pressing need to build a system that runs smoothly without being centralized. But this is fundamentally fallacious as the economic system within which is trying to be established itself as a dominant currency is hostile to decentralized systems. Given that the existence of bitcoin creates new profit opportunities then bitcoin is clearly a form of displacement. All in all, it is hard to consider of any other asset that clearly disproves the labour theory of value than bitcoin which has no physical manifestation.

The current economic environment of accommodative monetary policy and zero-low-bound interest rates is conducive to higher expected return, thus higher speculative behaviour which effectively leads to a euphoric state. Bitcoin's value is ultimately determined by the subjective valuation of 'investors' that is what the marginal buyer is willing to pay for under the fear of 'missing out' the market. The underlying enthusiasm feeds more demand for bitcoin driving expectations even higher. The short-lived initial euphoria paves the way to a more pessimistic outlook which causes a sharp price fall, crashes and ultimately panic.

\section{Conclusions}

The plethora and adoption of cryptocurrencies have taken off and the crypto-industry which has become more fluid, makes the lines between exchanges and wallets blurrier. Meanwhile, the absence of a secure and 
effective regulatory framework poses questions on the viability of the entire ecosystem designed to support the multitude of emerging virtual currencies.

In this study, we argue that the evidence of a speculative bubble in the price of bitcoin and its volatile nature does undermine its primary function as a means of exchange. Unfortunately, the frenzy speculation in bitcoin brings back to memory the dark side of financial innovations that tend to spring up frequently in an economic system permeated by highly volatile economic activity.

Certainly, the bitcoin along with other cryptocurrencies has spurred a revolution in payment technology that, if treated cautiously can facilitate financial intermediation and inclusion. Nonetheless, the price volatility and swings in trading volume and the evidence so far suggest that bitcoin displays features associated with speculative bubbles that can be explained by the mainstream explanations of irrational exuberance, manias or bubbles by Kindleberger (1978) combined with the heterodox approaches related to Minsky's (1992) financial instability hypothesis. These works are particularly relevant in the bitcoin's case as they provide a framework of analysis in predicting future episodes of economic contraction.

The heterodox perspective on the causes of financial crises can be, to a certain extent used to in bitcoin's case as well to provide a general framework of analysis in predicting future episodes of economic contraction. In our view, the financial complexity that has been precipitated by cryptocurrencies can only be dealt with by the development of an effective regulatory framework, a stable international lender of last resort (Kindleberger, 1978). Until then, the only certainty about the future of bitcoin is a substantial degree of uncertainty (Lo and Wang, 2014) and increasing regulatory risks which provide more room for speculation at the expense of its primary role as a means for electronic cash payments (Nakamoto, 2008). Ultimately, whether or not bitcoin constitutes a bubble is a decision for investors. The road to hell is paved with good promises. 


\section{REFERENCES}

C. BAEK - M. ElBECK (2014), "Bitcoins as an Investment or Speculative Vehicle?", A First Look Applied Economic Letters, 22 (1), pp. 30-34.

D. G. Baur - A. D. Hong - A. D. Lee (2017), "Bitcoin: Medium of Exchange or Speculative Assets?", Journal of International Financial Markets, Institutions \& Money (2017), https://doi.org/10.1016/j.intfin.2017.12.004

J. Bouoiyour - R. Selmi (2015), "What Does Bitcoin Look Like?", Annals of Economics and Finance, 16 (2), pp. 449-492.

J. Bouoiyour - R. Selmi - A. K. Tiwari (2015), "Is Bitcoin Business Income or Speculative Foolery? New Ideas Through an Improved Frequency Domain Analysis", Annals of Financial Economics, 10 (1), pp. 1-23.

I. CASPI (2017), "Rtadf: Testing for Bubbles With Eviews", Journal of Statistical Software, 81, pp. 1-16.

E.-T. CHeAH - J. Fry (2015), "Speculative Bubbles in Bitcoin Markets? An Empirical Investigation into the Fundamental Value of Bitcoin”, Economic Letters, 130, pp. 32-36.

J. H. Cochrane (2017) Bitcoin and bubbles. Available at: https://johnhcochrane. blogspot.gr/2017/11/bitcoin-and-bubbles.html

D. Fantazzini - E. Nigmatullin - V. Sukhanovskaya - S. Ivliev (2016), "Everything You Always Wanted to Know About Bitcoin Modelling But Were Afraid to Ask", Applied Econometrics, 44, pp. 5-24.

J. E. Gomez-Gonzalez - J. A. Parra-Polania (2014) "Bitcoin: Something Seems to Be 'Fundamentally' Wrong”. Bank of Colombia Working Paper.

A. Haldane (2017) Everyday Economics. Speech delivered at Nishkam High School, Birmingham on November 27, 2017.

D. He - K. Habermeier - R. Leckow - V. Haksar - Y. Almeida - M. Kashima - N. Kyriakos-SaAd - H. Oura - T. S. Sedik - N. Stetsenko - C. Verdugo-Yepes (2016) Virtual Currencies and Beyond: Initial Considerations. IMF Staff Discussion Note.

J. D. Hamilton (1986), On testing for self-fulfilling speculative price bubbles. International Economic Review, 27(3), pp. 545-552.

C. Kindleberger (1973), The World in Depression, 1929-1939. Berkeley: University of California Press.

C. Kindleberger (1978), Manias, Panics and Crashes: A History of Financial Crises. New York: Basic Books.

J. Kregel (2014), "Minsky and Dynamic Macroprudential Regulation", PSL Quarterly Review, 67 (269), pp. 217-238.

A. Kroeger - A. Sarkar (2017) 'The Law of One Bitcoin Price?", Federal Reserve Bank of New York, Working Paper.

S. Lo - J. C. WANG (2014) Bitcoin as Money? Federal Reserve Bank of Boston, Current Policy Perspectives.

A. MacDonell (2014) Popping the Bitcoin Bubble: An Application of LogPeriodic Power Law Modeling to Digital Currency. Preprint.

A. Malhotra - M. Maloo (2014) Bitcoin-Is it a Bubble? Evidence From Unit Root Tests (March 1, 2014). Available at: https://ssrn.com/abstract=2465891 
H. P. Minsky (1975), John Maynard Keynes. New York: Columbia University Press.

H. P. Minsky (1986) Stabilizing an Unstable Economy. Hyman P. Minsky Archive. Paper 144. http://digitalcommons.bard.edu/hm_archive/144

H. P. MinsKY (1992) "The Financial Instability Hypothesis. The Jerome Levy Economics Institute of Bard College”, Working Paper 72, May 1992.

H. P. Minsky - C. CAmbell (1987), "How to Get Off the Back of a Tiger, Or, Do Initial Conditions Constrain Deposit Insurance Reform?", in Merging Commercial and Investment Banking-Risks, Benefits, Challenges: Proceedings, A Conference on Bank Structure and Competition, ed. by Federal Reserve Bank of Chicago. Chicago: Federal Reserve Bank of Chicago, pp. 252-266.

S. NAкамото (2008) Bitcoin: A Peer-to-Peer Electronic Cash System. Available at: http://nakamotoinstitute.org/literature/

P. Phillips - T. Magdalinos (2007), "Limit Theory for Moderate Deviations from a Unit Root", Journal of Econometrics, 136 (1), pp. 115-130.

P. PhILliPs - Y. Wu - J. Yu (2011), "Explosive Behaviour in the 1990s NASDAQ: when Did Exuberance Escalate Asset Values?", International Economic Review, 52 (1), pp. 201-226.

P. Phillips - S. Shi - J. Yu (2015), "Testing for Multiple Bubbles: Historical Episodes of Exuberance and Collapse in the S\&P 500", International Economic Review, 56 (4), pp. 1043-1077.

J. SCHUMPETER (1934), Theory of Economic Development: An Inquiry into Profits, Capital, Credit, Interest, and the Business Cycle, Cambridge, MA: Harvard University Press.

D. Sornette - P. Cauwels (2014) "Financial Bubbles: Mechanisms and Diagnostics". Swiss Finance Institute Research Paper Series $N^{\circ} 14-28$.

D. YeRMACK (2014) "Is Bitcoin a Real Currency? An Economic Appraisal". NBER Working Paper No. 19747.

\section{Non-technical Summary}

In this paper, by using econometric techniques we provide evidence that bitcoin exhibited the formation of speculative bubble in 2017. To conceptually rationalize the results, we delve into the extant theoretical approaches developed by Kindleberger's (1978) speculative bubbles and Minsky's (1992) financial instability hypothesis. Certainly, bitcoin has spurred a revolution in payment technology that, if treated cautiously can facilitate financial intermediation and inclusion. Ultimately, whether or not bitcoin constitutes a bubble is a decision for investors as the road to hell is paved with good promises. 